\title{
IX. EPILOG
}

Anna d'Este, Herzogin von Nemours, Genevois und Chartres, Gräfin von Gisors, Dame von Montargis, Caen, Bayeux und Falaise, starb am späten Nachmittag des 17. Mai 1607 in ihrem Hôtel in Paris, an einer als Wassersucht bezeichneten Krankheit. Im November zuvor hatte sie ihr fünfundsiebzigstes Lebensjahr vollendet, und es war vor allem der Hinweis auf dieses hohe Alter, welcher in den Kommentaren der Zeitgenossen nicht fehlen durfte. So erfuhr man in Spanien, die Herzogin, la vieja, sei gestorben, und in dem nach Rom gesandten Bericht war ergänzt worden: finalmente'. Sechzig Jahre lang, unter fünf Königen aus zwei Dynastien hatte Anna in Frankreich gelebt, sie war Augenzeugin der großen Ereignisse der Zeit, und vielen mußte es scheinen, als sei sie schon immer dagewesen. Entsprechend gestalteten sich die Spekulationen über ihr wahres Alter: On la disait âgée de quatre-vingts ans; autres disaient soixante-dix-huit. Elle s'en donnait soixante-seize ${ }^{2}$. Ihr hohes Ansehen bei den Fürsten Europas ist aus den an ihren Sohn gerichteten Beileidsschreiben $\mathrm{zu}$ ersehen. Die Königin hob son mérite et le ranq qu'elle tenoit en ce royaume hervor, der Herzog von Lothringen schrieb, es sei bien raisonnable, qu'une princesse de sa qualité et de son mérite porte avec soy au tombeau les regretz de ceulx qui avoient l'honneur de luy attoucher, und Carlo Emanuele versuchte, mit dem Hinweis auf sa vie tant onorable et admirabel parmi le $[s]$ traverses de ce monde, et sa fin si glorieuse en un eage si avancé zu trösten ${ }^{3}$. Warme Worte kamen auch aus Italien, etwa von der Großherzogin von Toskana, die bezüglich der Dame, welche beinahe ihre Schwiegermutter geworden wäre, erklärte: elle avoit une prudence et valeur si grande, que vrayment je l'honnorois comme ma propre mère, während ihr Gemahl auf die Bedeutung der Prinzessin nicht nur für ihre eigene Familie, sondern für die allgemeinen Belange der Zeit hinwies:

La signora duchessa, madre di V.E., era principessa di tanto singolar bontà e prudenza, che la larghezza della sua vita importava allo interesso publico, non che al privato della persona

1 Diego de Lizárraga an Philipp III., 21. Mai 1607, Paris, in: AN, 21 Mi 63; Avviso aus Paris, (29.) Mai 1607, in: ASV, Segr. Stato, Francia 51, fol. 328r. Der Hinweis auf eine lente hydropisie als Todesursache: LA PALUD, Discour funebre, S. 24.

${ }^{2}$ L'ESTOILE, Journal Henri IV, Bd. 2, S. 242 (17. Mai 1607).

${ }^{3}$ Maria von Medici aus Fontainebleau, Charles de Lorraine aus Nancy, Carlo Emanuele di Savoia aus Turin an Henri de Savoie, 18. Mai, 15. Juni, 15. Juli 1607, in: BnF, Fr. 3647, fol. $116,120,126$. 
e casa dell'E.V., et ho più volte sentito affirmare, che in corte era ammirata e reverita come specchio di modèstia, di saviezza e di gravitát.

Den größten Verlust verspürten aber wohl die in Annas Diensten stehenden Personen, welche nicht nur eine Fürstin verloren hatten, für die sie tătig gewesen waren, manche sogar jahrzehntelang, sondern einige von ihnen auch ihre finanzielle Sicherheit und die Möglichkeit, Söhnen, Töchtern oder jüngeren Geschwistern eine Anstellung zu verschaffen. Dies bringt Antoine Favre zum Ausdruck, einer der Päsidenten des Herzogtums Genevois, welcher sich zuvor furr Annas Prozesse mehrere Male nach Italien begeben hatte:

La perte, à la verité, est commune à tous ceux qui avoient l'honneur d'estre en son service. Mais elle n'est pourtant egale à tous, et si je ne me flatte en mon mal je doy croire, ce me semble, que j'y ay perdu plus que nul autre, pour la grace plus particulière qu'elle me faisait de m'aymer et tous les miens'.

\section{Herz und Eingeweide - Der Körper der Toten}

Per Testament hatte Anna genaue Anweisungen gegeben, was nach ihrem Tod mit ihren sterblichen Überresten geschehen solle und wo sie beigesetzt werden wollte. Daraus sind vor allem zwei Dinge zu ersehen: Die Angst der Prinzessin, ihr Korper müsse geöffnet werden, und ihre Zugehörigkeit zu zwei Ehemănnern und deren Häusern:

Je laisse mon corps à la terre don[t] il est procéddé, et après estre morte je déffende d'estre ouverte, si ce n'estoit que l'on ne peult porter mon corps sans l'ourvir à Nyssy [Annecy], là où je veubx estre portée et ensépulturée au mesme lieu et en la mesme cave et dans la mesme sépulture là où l'est Monseigneur Jacques de Savoye mon mary, duc de Nemours et de Genevois, et notre fils Charles Emanuel, duc de Nemours, et notre fille Marguerite. Je veulx, $s$ 'il est possible sans $m$ 'ourrir, que l'on me fende le costé, mais que l'on attende vingt quatre heures après ma mort, et que l'on preigne mon cour et que l'on le mecte en l'une cassette de plomb et que l'on le porte à Joynville et qu'il soit mis tout contre le corps et cereveil de feu Monsigneur Francoys de Lorrayne mon premier mary, duc de Guyse. Et si d'avanture l'on ne pouvait faire tout cecy sans que je fusse ouverte, je veulx et commande que ce soit secrèttement et qu'il n'y ait poinct d'hommes, horsmis les chirurgiens nécessaires et en plus petit nombre que l'on pourra, et de mes femmes deux des plus vielles ${ }^{6}$.

${ }^{4}$ Christine de Lorraine und Ferdinando de' Medici an Henri de Savoie, 28. u. 30. Juli 1607, Florenz, in: BnF, Fr. 3647, fol. 130r, 138r. Vgl. die Briefe der Herzöge von Modena, Urbino, Mantua u.a., in: Ibid. u. Fr. 3650.

${ }^{5}$ Antoine Favre an Henri de Savoie, 9. Juni 1607, Annecy, in: BnF, Fr. 3647, fol. 117r.

${ }^{6}$ Testament Anna d'Este (fol. 98r). 
Sich in seine Einzelteile zerlegen und an verschiedenen Orten beerdigen zu lassen war nicht ungewöhnlich. Seit Beginn des 14. Jahrhunderts wurden Herz und Eingeweide aller französischen Könige getrennt von ihren Körpern beigesetzt, und der Adel imitierte diese Praxis. Während der Körper meist in der Hauptgrabstätte ruhte, konnte das Herz an einem für den Verstorbenen wichtigen Ort oder gemeinsam mit dem eines Freundes oder Vertrauten beigesetzt werden. So ließ Karl V. seinen Körper in Saint-Denis, sein Herz in Rouen, der Hauptstadt seines Herzogtums Normandie, seine Eingeweide nahe des Grabes seiner Mutter beerdigen. Die Herzen von Heinrich II. und Anne de Montmorency wurden zusammen in der Kirche des Pariser Cölestinerklosters beigesetzt, während das Herz Heinrichs IV. fern des Körpers in einer Kapelle in La Flèche ruht. René d'Anjou, Titularkönig von Sizilien, wollte 1480 in der Familiengrabstätte der Kathedrale von Angers beerdigt werden, wăhrend Herz und Eingeweide an anderen Orten beigesetzt wurden. Seine Gemahlin Jeanne de Laval tat es ihm nach: Ihr Körper ruht gemeinsam mit dem Körper des Gemahls, ihr Herz gemeinsam mit seinem Herzen ${ }^{7}$.

Zu Lebzeiten verfaßte René d'Anjou das "Livre du Cuer d'Amours espris«, in dem das Herz in Form des Ritters Cuer eine zentrale Rolle spielt. Obwohl es sich bei diesem allegorischen Roman um die Geschichte einer Liebeserfahrung handelt, das Herz also als Sammelpunkt der Emotionen eines Menschen verstanden wird, darf man die Tatsache, daß Anna d'Este ihr Herz nahe dem Körper ihres ersten Gemahl wissen wollte, nicht als Hinweis auf ihre swahren Gefühle oder 'wirklicher Liebe mißdeuten. Laut kanonischem Recht wurde der Körper einer Frau, die zu Lebzeiten mehr als einmal verheiratet war, im Grab ihres letzten Ehemannes beigesetzt; die Ruhestätte ihres Herzens war jedoch nicht festgelegt ${ }^{8}$. Der wohl einem Künstler der Schule von Fontainebleau zuzuschreibende und heute im Louvre aufbewahrte Entwurf eines gemeinsamen Grabmals der Herzen von Anna und François läßt indes die Vermutung zu, daß die Prinzessin ursprünglich sogar eine Vereinigung ihrer beiden Herzen geplant hatte, entweder in Notre-Dame in Paris, wo das Herz ihres Gemahls ruhte, oder aber in Joinville. Das Vorhaben muß sich jedoch als undurchfuhrbar erwiesen haben, da der Entwurf nie ausgefuhrt und Annas

${ }^{7}$ Ralph E. GIESEY, The Royal Funeral Ceremony in Renaissance France, Genf 1960, S. 19 22. Zu Karl V., René d'Anjou und Jeanne de Laval: Françoise ROBN, Quelques remarques sur l'art funéraire à la cour du roi René: de l'enfeu au sarcophage à l'italienne, in: Le roi René. René, duc d'Anjou, de Bar et de Lorraine, roi de Sicile et de Jérusalem, roi d'Aragon, comte de Provence, 1409-1480, Avignon 1986, S. 158-173, hier S. 161. Zu Heinrich II.: Brigitte BEDOS REZAK, Anne de Montmorency, seigneur de la Renaissance, Paris 1990, S. 257. Zu Heinrich IV.: BABELON, Henri IV, S. 986.

8 René D'ANJOU, Vom liebentbrannten Herzen, hg. v. Franz UNTERKIRCHER, Graz 1975; Michel SIMONIN, L'ouverture de la terre, in: Jean BALSAMO $(\mathrm{Hg}$.), Les funérailles à la Renaissance. XII colloque international de la Société française d'étude du seizième siècle, Genf 2002, S. 15-33, hier S. 21. 
Herz, wie im Testament bestimmt, neben dem Körper des Herzogs beigesetzt wurde?.

Den Leichnam der Prinzessin ohne vorherige Einbalsamierung nach Savoyen zu schaffen war natürlich nicht möglich, doch wurde die testamentarisch gebotene Wartezeit eingehalten. Am späten Abend des auf ihren Tod folgenden Tages wurden Annas Eingeweide im Chor der Kirche des Augustinerkonvents nahe dem Hauptaltar beigesetzt, asisté [de] grand nombre de noblesse, tant seigneurs que dames. Fünfundzwanzig Jahre später sollten neben denen der Mutter die Eingeweide ihres jüngsten Sohnes beerdigt werden, und das Grab mit seinem schlichten Stein und der Aufschrift $C y$ dessoubs reposent les entrailles de feue tres haulte et tres puissante princesse madame Anne d'Est blieb erhalten, bis das Kloster Ende des 18. Jahrhunderts zerstört wurde ${ }^{10}$. Während in Saint-André-des-Arts noch eine große Messe für die Verstorbene gefeiert wurde, befand sich ihr Herz schon auf dem Weg nach Joinville, wo es in der Gruft der zum Schloß gehörenden Kapelle Saint-Laurent beigesetzt wurde. Auch diese Gräber wurden Anfang der 1790er Jahre zerstört und die sterblichen Überreste der Herzöge in ein Massengrab geworfen, kurz darauf aber wieder ausgegraben und auf dem öffentlichen Friedhof der Stadt beigesetzt ${ }^{11}$.

Dem Wunsch der Prinzessin entsprechend wurde ihr Körper nach Savoyen gebracht, begleitet von ihrem maitre d'hôtel, Camille Mahy, und den meisten Herren ihres Gefolges, darunter auch ihr Almosenpfleger Nicolas Voilard. Anfang Juni langte der Zug in der Umgebung von Annecy an, und die Tote wurde in der Kirche des Klosters von Sillingy aufgebahrt, umgeben von brennenden Kerzen und bewacht von den Priestern, welche ohne Unterlaß Gebete sprachen $^{12}$. Drei Tage blieb die Tote in der Klosterkirche, bis sie am Abend

${ }^{9}$ Louvre, Cabinet des dessins, Inv. Nr. 8830. Eine Abb. in: Ian WARDROPPER, Un projet de monument aux cœurs de François de Lorraine et d'Anne d'Este, in: Yvonne BELLENGER (Hg.), Le mécénat et l'influence des Guise. Actes du Colloque de Joinville 1994, Paris 1997, S. 279-291, hier S. 291. Während Sylvie BÉGUIN, L'École de Fontainebleau: des »Histoires anciennes... et modernes«, in: Roberto CASSANELLI ( $\mathrm{Hg}$.), Ateliers de la Renaissance, SaintLéger-Vauban, Paris 1998, S. 275-296, hier S. 277, den Entwurf Primaticcio zuschreibt, plädieren WARDROPPER, Projet de monument, und Dominique CORDELLIER, Entourage de Primatice, Monument mural des cœurs de François de Lorraine et d'Anne d'Este, in: Primatice. Maître de Fontainebleau, Kat., Paris 2004, S. 469f., für einen Künstler aus seinem Umkreis. Das Wappen, aufgrund dessen Anna als Auftraggeberin für das Grabmal identifiziert wurde, ist gespalten und zeigt links tatsächlich einen Adler, das Wappentier der Este. Welche Familie oder Person rechts repräsentiert sein soll, ist unklar.

${ }^{10}$ L'EsToILE, Journal Henri IV, Bd. 2, S. 242 (18. Mai 1607); Émile RAUNiÉ (Hg.), Épitaphier du vieux Paris, Bd. 1: Saint-André-des-Arcs - Saint-Benoit, Paris 1890, S. 166.

${ }^{11}$ Extraits des registres de l'église, S. 481 (6. Juni 1607); Epitaph der Herren von Joinville, in: BouILLÉ, Histoire, Bd. 1, S. 548. Zum Schicksal der Gräber: Ibid. S. 231.

${ }^{12}$ BARFÉLY, procureur fiscal au Conseil présidial, Relation des funérailles faictes a Madame Anne d'Est, femme de Jacques de Savoye, duc de Genevois et de Nemours, qui fut inhumée dans l'Eglise de nostre-Dame d'Annecy, in: Claude-A. DucIS, Anne d'Este, duchesse de 
des 6. Juni nach Annecy gebracht wurde, begleitet von den Repräsentanten und den Bewohnern der Stadt, den Angehörigen des lokalen Adels en fort digne equipage et en plus grand nombre quon ne pensoit, den Mönchen der Klöster und Priestern der Kirchen, von fünfzig, zu diesem Zweck eingekleideten Armen mit Kerzen in den Händen, vor allem aber vom Genfer Bischof, dem später heiliggesprochenen Franz von Sales, der sich zu diesem Anlaß prachtvoll gekleidet hatte ${ }^{13}$. Der Sarg, bedeckt von einem schwarzen Tuch mit weißem Kreuz, wurde von vier Pferden gezogen, neben dem Wagen schritten vier Männer einher, die den Baldachin aus schwarzem Samt mit seidenen Fransen trugen, und trotz der Dunkelheit und des Regens schloß sich eine große Menge Volkes an, sans ordre toutefois et sans commandement autre que du zele quil auoient de rendre leur deuoir. Aufgrund der Anordnung des Herzogs von Nemours, die Beisetzungszeremonie am frühen Abend, entre jour et nuit, abhalten zu lassen, wurden die Teilnehmenden Zeuge eines prachtvollen Spektakels, denn dem Zug voran gingen dreihundert Fackelträger, und die Fenster der Häuser der Stadt waren mit so vielen brennenden Kerzen versehen, $\mathrm{da}$ es schien, als stünden die Straßen, durch die der Leichnam getragen wurde, in Flammen ${ }^{14}$.

Am Portal der Kirche Notre-Dame-de-Liesse angekommen, an welchem Annas Wappen und das Motto Speculum patientiae angebracht waren, wurde der Sarg in den mit schwarzem Tuch bespannten Chor getragen und die Tote auf einem Katafalk aufgebahrt, der von brennenden Kerzen umgeben war. Die Beerdigungszeremonie fand erst am nächsten Tag statt und bestand aus drei hintereinander gelesenen Messen sowie der anschließenden Leichenrede des Bischofs; am Abend wurde der Körper der Toten neben denen ihres Gemahls und ihrer beiden Kinder beigesetzt. Jeden Samstag betete man in der Kirche für die Verstorbenen, und einmal im Monat wurden die Glocken à forme de lamentations geläutet. Noch Ende des 17. Jahrhunderts war die Grabstätte mit einem schwarzen, mit goldenen Fäden bestickten Samttuch bedeckt, im Juli 1793 wurde sie zerstört und die Gebeine der Fürsten auf dem städtischen Friedhof beigesetzt. In dem heutigen, aus dem 19. Jahrhundert stammenden Kirchengebäude erinnert eine Gedenktafel an diejenigen, welche einst in Notre-Dame-de-Liesse ihre letzte Ruhestätte fanden; die Prinzessin aus Ferrara ist außerdem auf einem in der ersten Hälfte des 20. Jahrhunderts installierten Glasfenster zu sehen, wie sie, gemeinsam mit ihrem Gemahl, das Heilige

Genevois et de Nemours. Ses obsèques à Annecy en 1607, Annecy 1891, S. 10-27, hier S. 14. Zu den die Tote nach Savoyen begleitenden Personen: Anhang A, fol. 3r.

${ }^{13}$ BARFÉLY, Relation des funérailles, S. 19f. Vgl. den 32 Namen verzeichnenden "Roolle des gentilzhommes de Foucigny qui ont assité au dueil et obsèques de Madame«, o.D. (Juni 1607), in: AST (Cast.), Int., PGN, cat. 2, m. 14, n. 20.

${ }^{14}$ Register der Beschlusse des Stadtrats von Annecy, 2. Juni 1607, in: AMA, BB 11, vol. 29, fol. 231r; BARFÉLY, Relation des funérailles, S. 16f., $21 \mathrm{f}$. 
Leichentuch anbetet, welches im Sommer 1566 aus Anlaß ihres Einzuges in die Stadt aus Chambéry nach Annecy gebracht und ausgestellt worden war ${ }^{15}$.

\section{Königin und Heilige - Strategien der Erinnerung}

Das Begräbnis der Herzogin von Nemours wäre einer Königin wlirdig gewesen, und tatsächlich wollte ihr Sohn genau diesen Eindruck erwecken. Seine Untertanen sollten um die Mutter trauern, als wäre sie die Herrscherin eines souverănen Staates, oder besser noch, eine Heilige. In einem Schreiben Henris an den Stadtrat von Annecy war Anna daher kurzerhand zur Beschlitzerin des Ortes erklärt worden:

vous luy estes fort obligés de l'honneur quelle vous a fait choisissant en nostre ville de Nissy sa sepulture, car un plus grand signe d'amour ne vous pouvoit elle monstrer que de vouloir que ses cendres de si loing vous soient portees pour reposer aupres de vous [...], en recevant ces honorables reliques, vous en soyez recompensez a l'advenir car de la hault vous regardant selon vos bonnes intentions elle aura soin de vos personnes et familles et fera par ses prieres que les benedictions de Dieu ne vous defauldront point ${ }^{16}$.

"Glorreich« ist folglich das Wort, welches sich am häufigsten in den Beschreibungen der Bestattungszeremonien findet: Die Stadtväter fühlten sich glorieux de l'honneur que leur auoit fait Madame de donner son Corps a leurd uille, und die Männer und Frauen aus dem einfachen Volk bezeugten mit ihren Tränen leur affection envers la glorieuse memoire de Madame ${ }^{17}$. Der daraus entstehende Nutzen furr Henris Ansehen war groß, denn nicht nur die eigenen Leute, auch die in Savoyen stationierten spanischen Truppen zeigten sich beeindruckt. Die Zeremonien seien nicht nur zur Zufriedenheit der Bevölkerung von Annecy verlaufen, wie der Präsident Favre dem Herzog versicherte, sondern auch zu der der Spanier, qui ont dit tout haut et advoué que si

\footnotetext{
${ }^{15}$ Mémoire historique sur la ville d'Annecy, fol. 62vf.; François-Luc de Lucinge, Estat des provinces de Genevois et Foucigny, 1691, zit. in: Annesci 12 (1965) S. 58; Max BRUCHET, Jacques de Savoie, duc de Genevois-Nemours. Instruction et Discours sur le faict du Gouvernement (1582), in: Revue savoisienne 39 (1898) S. 103-130, 178-205, 284-289, hier S. 110, Anm. 4; Claude-A. DUCIS, Le poète Nouvellet, chanoine d'Annecy. La maison de Genevois-Nemours, in: Revue savoisienne 22 (1881) S. 109-111, 117-120, hier S. 119, Anm. 5; DERS., Le Saint Suaire à Annecy et la naissance de saint François de Sales, in: Revue savoisienne 24 (1883) S. 33-35.

${ }^{16}$ Henri de Savoie an den Stadtrat von Annecy, 22. Mai 1607, Paris, in: Ducis, Henri de Savoie, S. 41.

${ }^{17}$ BARFÉLY, Relation des funérailles, S. 15, 20.
} 
c'eust esté pour le Roy d'Espagne (qu'est le plus grand mot qu'ilz puissent dire en leur langage) on n'auroit rien peu faire de plus ny de mieux ${ }^{18}$.

Es wäre interessant, die Leichenrede, welche Franz von Sales der Verstorbenen gehalten hat, einer naheren Betrachtung zu unterziehen, denn gelobt wurde sie nicht nur als schön und elegant, sondern auch als si pleine pour ne rien oublier des principales louanges deües a la tres heureuse memoire de Madame, que toute l'Audience en demeura non seulement tres satisfaite mais rauie, doch leider ist sie nicht erhalten ${ }^{19}$. Der Bischof scheint seine Ansprache größtenteils improvisiert zu haben, denn er kam in Verlegenheit, als Henri ihn um eine Kopie des Textes bat: Monsieur de Nemours m'a tellement conjuré de luy envoyer l'Orayson funebre de Madame sa mere, que je suis contraint d'en escrire une presque tout autre; car je ne me resouviens pas de celle que je dis, sinon sgrosso modor. Noch im Herbst wartete er auf verschiedene memoires de la grandeur de la mayson d'Este aus Italien, denn aus den ihm zur Verfugung stehenden Werken habe er nichts erfahren können, das esclattant wäre, doch damit nicht genug: a la verité, je n'ay rien sceu des actions particulieres de cette Princesse ${ }^{20}$.

In der Regel unterstreichen französische Leichenpredigten vom Beginn des 17. Jahrhunderts die stoizistische Haltung des Verstorbenen, und sie rufen den Zuhörern Tod und Verlust in Erinnerung. So kann von einer vergleichbaren Rede möglicherweise auf die des Franz von Sales geschlossen werden. Im Juli 1607 wurde in der Kirche von La Ferté-Bernard, die zu Annas douaire gehörte, eine Leichenfeier abgehalten, in deren Rahmen der dortige Priester, Séverin Bertrand, eine Ansprache hielt. Hinsichtlich seines Stils ist dieser Vortrag sicher nicht annăhernd so elegant und gewandt wie es der des Bischofs gewesen sein muß, aber immerhin wurde das kleine Werk gedruckt und zirkulierte zwei Wochen später bereits in Paris. Nicht anders als sein Kollege aus Savoyen es vorhatte, begann Bertrand seine Ausführungen mit den »eklatanten« Tatsachen der Geschichte der Este, die in erster Linie in ihrem altehrwürdigen Herkommen und den Verbindungen $\mathrm{zu}$ anderen Häusern des europäischen Hochadels bestünden. Die Familie sei nicht nur si ancienne et illustre, daß die Historiker ihren Ursprung bis auf das Jahr 402 zurückverfolgen könnten, sondern auch mit sechs Kaisern, funfzehn Königen und dreißig Herzögen verschwägert. Hinsichtlich Annas persönlicher Verdienste hob der Priester ihren Glauben und ihre Gottesfurcht hervor, denn sie habe au mirouër d'elle-mesme

\footnotetext{
${ }^{18}$ Antoine Favre an Henri de Savoie, 9. Juni 1607, Annecy, in: BnF, Fr. 3647, fol. 117v.

19 Antoine Favre an Henri de Savoie, 22. Juni 1607, Annecy, in: PolzAT, Princesse de Clèves, S. 184; MUGNIER (Hg.), Petites Annales, S. 41; BARFÉLY, Relation des funérailles, S. 25.

${ }^{20}$ Franz von Sales an Jeanne de Chantal und Antoine des Hayes, 16. Aug. u. 12. Okt. 1607, Annecy, Les Clefs, in: Saint François de SALES, evêque et prince de Genève et docteur de l'église, Guvres, Bd. 13: Lettres, T. 3, Lyon, Paris 1904, S. 311 , 325.
} 
den wahren Gott erkannt und inmitten von grandeurs et richesses den eigenen Interessen entsagt: Car ce qui regardoit son profit particulier elle a negligé: mais en ce qui estoit de l'honneur de Dieu et du public, elle s'est ouverte et eslargie ${ }^{21}$.

Dies ist die einzige Stelle, an der Bertrand auf die Bemühungen der Prinzessin um den honneur public zu sprechen kommt, ansonsten unterscheidet sich seine Rede nicht sonderlich von jenen zu Ehren anderer Fürstinnen der Zeit. $\mathrm{Da} B$ ăhnliches auch für außerhalb Frankreichs entstandene protestantische Funeralwerke gilt, zeigt das Beispiel der 1585 verstorbenen Anna von Dänemark, Kurfürstin von Sachsen. In den zu ihrem Andenken verfaßten Leichenpredigten tritt das Biographische ebenfalls hinter dem Lob ihrer christlichen Tugenden zurück, nicht anders als in Bertrands Darstellung des Lebens der Anna d'Este: L'humilité, la pieté, la modestie, la magnanimité, et autres divines qualitez de son ame werden gelobt, ihre Taten verglichen mit denen einer Judith, einer Esther oder Blanche, Mutter Ludwigs des Heiligen, ihre letzten Stunden und ihr Tod mit denen der heiligen Paula. Schließlich wird ihr Wesen zu einer »ewigen Lilie« verklärt, denn wie die Lilie alle anderen Blumen übertreffe, so sei auch Annas Seele glücklicher als die der anderen: $C$ 'est l'ame de nostre Princesse, qui n'est point morte. Mit göttlichen Tugenden reich versehen, beinahe wie eine Heilige, soll sich die Welt der Herzogin von Nemours erinnern, und Bertrand schließt seine Ansprache mit den Worten: Toutes ces divines vertuz graveront son nom en la memoire des siecles, et elle regnera pour jamais. Der gedruckten Fassung der Rede ist eine Inschrift für das Grabmal der Prinzessin beigegeben, welche den Vorübergehenden auffordert: Viator siste viam, obviam veni: haec huius defunctae profero laudi, audi nomen et omen, die jedoch nie auf einem wirklichen Grabstein zu lesen war $^{22}$.

Es war dies nicht die einzige anläßlich von Annas Tod entstandene literarische Schöpfung, viele weitere Stücke in Versen und in Prosa, auf Latein und Französisch sollten in den folgenden Monaten verfaßt werden, und selbst die Mitglieder der kurz zuvor in Annecy gegründeten Académie Florimontane wurden aufgefordert, [de] faire quelque chose de bon sur un si fâcheux sujet ${ }^{23}$.

${ }^{21}$ BERTRAND, Oraison funebre, fol. 4r, 10v, 13r, 16v. Zur Verbreitung der Schrift in Paris: L'ESTOLE, Journal Henri IV, Bd. 2, S. 262 (27. Juli 1607). Zur französischen Leichenpredigt des 17. Jh.: Bernard VOGLER, Leichenpredigten und die aktuellen Forschungen der französischen Historiographie, in: Rudolf LENZ (Hg.), Leichenpredigten als Quelle historischer Wissenschaften, Köln, Wien 1975, S. 200-208, hier S. $200 \mathrm{f}$.

${ }^{22}$ BERTRAND, Oraison funebre, fol. 16r-v, 17v. Zu Anna von Dänemark: Jill BEPLER, nim dritten Gradu ungleicher Linie Seitwarts verwandt . Frauen und dynastisches Bewußtsein in den Funeralwerken der Frühen Neuzeit, in: Heide WUNDER (Hg.), Dynastie und Herrschaftssicherung in der Frühen Neuzeit. Geschlechter und Geschlecht, Berlin 2002, S. 135-160, hier S. 139-141.

${ }^{23}$ Antoine Favre an Henri de Savoie, 22. Juni 1607, Annecy, in: PolZAT, Princesse de Clèves, S. 185. Das Wort in Klammem wurde von mir anhand BnF, Fr. 3647, fol. 122r korrigiert. 
So erschien in Chambéry zu Beginn des Jahres 1609 eine Leichenrede, deren Verse um das Thema der Patientia kreisten, eine Tugend, mit der die Prinzessin auf so außerordentliche Weise begabt gewesen sei, daß sie den Zorn der Fortuna hervorgerufen habe. Diese habe nämlich über Annas Haupt zeitlebens un deluge de maux, une mer de malheurs, un monde de travaux niedergehen lassen, die mit einigen Seufzern, ansonsten aber nur mit grave constance beantwortet worden seien - die übernatürliche Geduld der Prinzessin konnte folglich allein von den Parzen beendet werden. Auch in dieser Schrift wird die Prinzessin zu einer Göttin erklärt, die von ihrer himmlischen Wohnstätte aus denjenigen beistunde, die sie um Unterstützung gegen die Machenschaften der Fortuna baten: Ores ell' est au ciel tenue pour Deesse, / Deesse qui pourra ceux qui l'imploreront, / Deslivrer des ennuicts que tes traictz leurs feront ${ }^{24}$.

\section{Testament und Hinterlassenschaft}

Anna hat ein Testament verfaßt, das ist sicher, denn ein zum Zeitpunkt ihres Todes im Hôtel de Nemours vorhandener Entwurf wird im Inventar folgendermaßen beschrieben: un testament de lad. deffuncte dame de Nemours, escript et signé de sa main [...], estant en deux feuilletz de papier, escriptz sans jour ny datte, [...] signé en fin ANNE D'EST ${ }^{25}$. Entstanden ist dieser Entwurf frthestens Ende 1597, an einer Stelle ist von Annas procès d'Italie die Rede, der nach dem Tod des Bruders Alfonso begonnen hatte. Die Prinzessin scheint jedoch nie einen Notar herangezogen zu haben, um ihren letzten Willen zu beglaubigen, denn in den Akten der von ihr normalerweise konsultierten Notare ist kein von ihrer Hand unterzeichnetes Testament vermerkt, und es sind auch keine Abschriften davon in den Archiven von Modena und Turin zu finden. Von ihrem Entwurf blieb nur eine einzige, Jahrzehnte später entstandene Kopie von kaum zweieinhalb Seiten erhalten, welcher offensichtlich ein ganzer Teil fehlt, und es ist unmöglich festzustellen, ob bereits das ursprüngliche Dokument unvollendet geblieben war oder ob es nur zur Hälfte abgeschrieben wurde. In dieser einzig vorhandenen Version ist, außer Annas zitierten Wünschen hinsichtlich ihrer Beisetzung, der Ernennung von Testamentsvollstreckern und den allgemeinen Anweisungen zur Ausführung, nur ein einziger Punkt ihres letzten Willens vermerkt: Je veux que les services qui se font par mon commandement à nostre dame de Saincte Claire de Nissy soient continués - direkt anschließend folgt die Kopie ihrer Unterschrift. Angesichts

${ }^{24}$ LA PALUD, Discour funebre, S. 6f., 26. Vgl. auch die lateinischen Verse von ClaudeÉtienne Nouvellet in: DucIS, Anne d'Este, S. $28 f$.

${ }^{25}$ Anhang A, fol. 29r. 
der vielen, während der letzten Monate und Wochen ihres Lebens getătigten Schenkungen zugunsten von weiblichen Angehörigen ihres Gefolges ist jedoch anzunehmen, daß Anna diese auch in ihrem Testament nicht vergessen hat $^{26}$.

Um die Ausfuhrung der einzelnen Klauseln zu garantieren und um zu verhindern, daß sich die Erben vor der Exekution ihres letzten Willens an ihrer Hinterlassenschaft bereichern könnten, bestimmte die Prinzessin, daß der gesamte Besitz zunächst in die Hände ihrer Testamentsvollstrecker ubergehen sollte, die dann ein Jahr Zeit hätten, ihren Anordnungen nachzukommen, ohne daß ihre Kinder oder Enkelkinder Zugriff auf ihr Erbe hätten. Daruber hinaus wollte Anna um jeden Preis verhindern, daß ihre Söhne und Enkel nach ihrem Tod ebensolche Streitigkeiten auszustehen und Prozesse gegen Mitglieder der eigenen Familie zu fuhren hätten wie sie selbst:

Et par ce qu'il a pleu à dieu et que sa volunté a esté telle que d'avoir retiré à soy huict de mes chers enfans, et qu'il ne m'a laissé de mon premier mariage que mon fils du Mayne et les enfans de mon fils de Guyse, et du second mon fils de Nemours, et que la chose du monde que je craindr[ois] et abhorrerois et regretterois le plus se seroit qu'ils vinsent en débat après ma mort pour si peu de bien que je laisse, je veulx et leur commande de se contenter de ma volunté ${ }^{2 f}$.

Am Tag nach Annas Tod versprach der Herzog von Nemours vor einem Notar, von seinen Rechten als Erbe keinen Gebrauch machen zu wollen und sich mit dem abzufinden, was die Mutter früher schon zu seinen Gunsten verfugt hatte. Tatsächlich konnte Henri zufrieden sein, denn im Sommer 1600 hatte die Prinzessin ihm nicht nur ihre Rechte am Herzogtum Nemours überlassen, sondern auch terre, seigneurie et forêt von Montargis sowie einige kleinere Besitztumer ${ }^{28}$.

Es war klar, daß die Herzöge von Mayenne und von Guise, letzterer das alteste Enkelkind der Verstorbenen, die Schenkung anzufechten gedachten, und bereits im Herbst 1607 kamen die Parteien zu einer Einigung, in deren Rahmen Annas Besitztümer oder die Einkünfte daraus unter den Erben aufgeteilt wurden. Der Herzog von Guise erhielt Montargis, welches er einige Jahre

${ }^{26}$ Testament Anna d'Este (fol. 99r). Bei den auf der Suche nach dem Testament in Paris konsultierten Akten handelt es sich um rund zwanzig zur Étude VIII des Minutier central des notaires parisiens gehörende Bände. Vgl. die den dames de Sainte Claire jährlich ausgezahlte Pension von über 573 Florins im Rechnungsbuch 1602 von Anna d'Este und Henri de Savoie für das Herzogtum Genevois, das Marquisat Saint-Sorlin sowie für Foucigny und Beaufort, in: AST (Cast.), Int., PGN, cat. 3, m. 1, n. 1. Zur Versorgung des Gefolges: Schenkung von 3000 l.t. von Anna d'Este an Madeleine Vaudelon, eine ihrer demoiselles, 2. Mai 1607, in: AN, m.c. VIII, 570, fol. 342 u. AN, Y 146, fol. 211v.

${ }^{27}$ Testament Anna d'Este (fol. 98v).

${ }^{28}$ Notarielle Erklärung von Henri de Savoie, 18. Mai 1607, in: AST (Cast.), Int., PGN, cat. 5 , m. 11, n. 6; Schenkung von Nemours, Montargis u.a. von Anna d'Este an Henri de Savoie, 28. Juli 1600, in: AN, Y 139, fol. 135r. 
spăter an den König verkaufte, Annas Sohn Charles die in der Normandie gelegenen Vicomtés Caen, Bayeux und Falaise und Henri Chartres, Gisors, das Nutzrecht an Gien sowie eine Summe von knapp 200000 Livres, wofür er sich allerdings verpflichtete, die Schulden der Mutter zu begleichen, ihren Bediensteten die ausstehenden Lơhne zu zahlen und die Kosten der Beerdigung zu tragen. In gemeinsamem Besitz verblieben die ausstehenden Schulden der französischen Krone beim Herzog von Ferrara und mithin bei Anna als dessen Erbin sowie alle durch den Tod Alfonsos entstandenen Ansprüche in Italien $^{29}$. Daß die drei Fürsten keineswegs bereit waren, die italienischen Forderungen aufzugeben, ist aus den Briefen zu ersehen, mit denen sie sich ein halbes Jahr später um die Unterstützung des Herzogs von Savoyen bemühten. Dessen Tochter sollte mit dem ältesten Sohn des Herzogs von Modena verheiratet werden, und Annas Erben waren der Überzeugung, Carlo Emanuele müsse daher großen Einfluß auf seinen zukünftigen Schwager haben und könne ihr gerechtes Anliegen vor Cesare besser vertreten ${ }^{30}$.

Hinsichtlich Annas beweglicher Hinterlassenschaft kam es, als diese im Hôtel de Nemours inventarisiert werden sollte, zu einer merkwürdigen Situation, denn es präsentierten sich nicht nur die rechtlichen Vertreter der Herzöge von Mayenne und von Guise, welche die Erbschaft nur unter Vorbehalt năherer Prufung anzutreten gedachten, sondern auch ein Abgesandter des Herzogs von Nemours. Dieser hatte zwar auf seinen Teil am Erbe verzichtet, war aber der Ansicht, da seine Eltern in ihrem Heiratsvertrag die Trennung ihrer Güter beschlossen hatten, Anspruch auf die Möbel und Wertgegenstände erheben zu können, welche sich ursprünglich im Besitz des Vaters befunden hatten, vor allem auf die Gemälde, einige Stücke des Tafelsilbers und einen Teil der Wäsche. Henris Vertreter Jean Vatebled griff daher mehrmals in die Inventarisierung ein und erklärte, besagte Bilder und Möbelstücke gehörten seinem Herm und dürften nicht inventarisiert werden, da sie aus dem Nachlaß von Jacques de Savoie stammten, woraufhin der Mann des Herzogs von Guise regelmaßig erwiderte, $q u$ 'il ne peult demourer d'acord ou dire dud. Vatebled ${ }^{31}$.

Viel wurde bei der Inventarisierung des Hôtel de Nemours nicht gefunden: Einige Kleinigkeiten, wie etwa drei Damespiele aus Ebenholz mit den dazugehörigen Spielsteinen, verschiedene Kistchen und Etuis aus Holz, Leder und

\footnotetext{
${ }^{29}$ Vertrag zwischen Charles de Lorraine, Herzog von Guise, Charles de Lorraine, Herzog von Mayenne, und Henri de Savoie, das Erbe von Anna d'Este betreffend, in: AST (Cast.), Int., PGN, 5. cat., m. 11, n. 7; Verschiedene Schriftstücke, den Verkauf von Montargis durch Charles de Lorraine, Herzog von Guise, und Henri de Lorraine, Herzog von Mayenne, an Ludwig XIII. betreffend, 17. u. 28. Febr. 1612, 7. Jan. 1613, in: LELOUP, Anne d'Este, S. 67-70.

${ }^{30}$ Charles de Lorraine, Herzog von Guise, und Charles de Lorraine, Herzog von Mayenne, an Carlo Emanuele di Savoia, 16. u. 18. Apr. 1608, in: AST (Cast.), Int., Let. div., Prin. for. 51.

${ }^{31}$ Als Beispiel: Anhang A, fol. 20r.
} 
Samt, siebenundzwanzig Fächer in unterschiedlichen Farben und Größen, ein Seidenschal, mit Perlen bestickt und mit einer Goldbordüre versehen sowie Taschen und Täschchen aus rotem, schwarzen und grüen Samt oder Seide, mit Gold- und Silberfäden oder mit Perlen geschmückt. Die Sammlung von Fläschchen und Ampullen, gefüllt mit verschiedenen Ölen, Parfüm und Pulvern, weist auf die Sorge der Herzogin für Körperpflege und Gesundheit hin und korrespondiert mit den in ihrem Gemach im Schloß von Lingotto vorhandenen Becken und Vasen per lavar le mani und per scaldar l'aqua sowie mit den monatlichen Ausgaben für den Apotheker, für Aderlässe und Bäder ${ }^{32}$. Wertgegenstände im eigentlichen Sinne sind im Inventar nicht in zu erwartender Menge verzeichnet. Die unter "meubles d'or, d'argent, orestal et autres" aufgezählten Teile sind wenige, bedenkt man den Schmuck und die Edelsteine, welche Anna einst aus Italien mit nach Frankreich gebracht und später an ihre Kinder weitergeschenkt hatte; insgesamt beläuft sich der Wert aller im Hôtel vorgefundenen Stücke auf weniger als tausend Livres. Hinzu kommt das Silber, welches sofort nach dem Tod der Prinzessin für siebenhundert Livres verkauft worden war, um die Außenstände bei Pariser Lebensmittelhändlern zu begleichen. Die kostbarsten der vorgefundenen Gegenstände stellen zwei Konfektdosen aus vergoldetem Silber dar, mit Porphyr und Halbedelsteinen besetzt, deren Wert zwar nicht berechnet wurde, für die aber mindestens zweihundertvierzig Livres veranschlagt worden sein müssen; außerdem ein aus siebzig Achatkugeln bestehender Rosenkranz und ein Diamantring, die beide mit einem Wert von je hundert Livres verzeichnet sind ${ }^{33}$.

Die im Hôtel de Nemours vorgefundenen kostbaren Stoffe nehmen mit ihrem fast die Hälfte des gesamten Vermögens ausmachenden Wert den wichtigsten Posten unter den verzeichneten Gegenständen ein. So wurden in einer mit rotem Satin ausgeschlagenen Truhe, für die gerade einmal fünfzig Sols veranschlagt waren, Stoffe im Wert von tausend Livres gefunden, darunter verschiedenfarbige, mit goldenen und silbernen Bordüren und kostbaren Stikkereien versehene Seide und holländische Spitze. Weitere achthundert Livres war die Wäsche wert, welche aus unzähligen Tüchern und Tüchlein bestand, aus Hemden, Kragen und Manschetten sowie Spitzenhalskrausen, aus Morgenröcken, Wamsen und den dazugehörigen Ärmeln, sogar aus einer der zu dieser Zeit selten anzutreffenden Unterhosen, aus seidenen Hauben und Häubchen und aus mehreren Stücken kostbarsten Kopfputzes, ouvrée d'or et de soye de plusieurs coulleurs ${ }^{34}$.

${ }^{32}$ Anhang A, fol. 8f.; Rechnungsbücher 1591, 1593-II. Die Zitate: Post-mortem Jacques, fol. 78r.

${ }^{33}$ Anhang A, fol. 13f., 19v. DUBOST, France italienne, S. 290, 293, berechnet fur die Wertgegenstănde eine Summe von 935 l.t., 14 s., was $21,8 \%$ des Gesamtwertes entspricht.

34 Anhang A, fol. 10f. Die Summe von 1941 l.t., 5 s. für die Stoffe entspricht $45,2 \%$ des Gesamtwertes der Hinterlassenschaft: DUBOST, France italienne, S. 290, 293. 
Was jedoch fehlte, waren Annas Kleider und Mäntel. Obwohl die Mitglieder ihres Gefolges aufgefordert wurden, alle veräußerten oder an sich genommenen Kleidungsstücke der Herzogin $\mathrm{zu}$ benennen, wurden nur wenige Teile aufgefunden. Einer der Kammerdiener gab an, einen Mantelsack aus schwarzem Tuch in Verwahrung gehabt zu haben, in dem sich ein kleiner, mit Marderpelz gefuitterter Mantel aus schwarzem Samt befand, sowie zwei weitere Mäntel, die er allesamt nach dem Tod seiner Brotgeberin für den Preis von vierundfünfzig Livres verkauft habe, außerdem tauchten zwei weitere Mäntel auf sowie ein Kleid, bestehend aus une cotte de camelot de turque, garnye de son corps de taffetas gris brun, le tout de peu de valleur. Es ist dies das einzige Kleid des gesamten Inventars, was hinsichtlich der Ausgaben, die Anna allein Anfang der 1590er Jahre für ihre Kleidung tätigte, erstaunlich ist. So wird in einem ihrer Rechnungsbücher ein Mantel aus Marderpelz aufgeführt, für den sie die exorbitante Summe von hundertzwanzig Écus bezahlte, de quoy y'ai eu bon marché, und bei dem es sich eventuell um das fünfzehn Jahre später für fünfundvierzig Livres verkaufte Teil handeln könnte ${ }^{35}$.

\section{Die Bibliothek des Hôtel de Nemours}

Kein anderer der im Inventar aufgefuhrten Posten ist so bemerkenswert wie die Bibliothek, bestehend aus hundertvierzig Einträgen; zăhlt man die in anonymen Paketen zusammengebundenen Bände mit, sogar fast zweihundert ${ }^{36}$. Hundert der Bücher sind mit ihrem Titel benannt, bedeutend weniger als in den Bibliotheksinventaren von Maria Stuart oder Katharina von Medici, aber beinahe genauso viele wie in dem dreißig Jahre zuvor angefertigten Inventar der Bibliothek des Dichters Rémi Belleau ${ }^{37}$. Die genannten Titel und Autoren machen deutlich, wo die Interessen der Prinzessin lagen, oder besser, wo sie im Laufe ihres Lebens gelegen haben, schließlich stellen die im Inventar ver-

\footnotetext{
${ }^{35}$ Anhang A, fol. 22vf.; Rechnungsbuch 1593-II (Juli, Sept.).

${ }^{36}$ Anhang A, fol. 23-28, hierauf beziehen sich auch die folgenden Ausführungen und Zitate.

${ }^{37}$ Die Inventare der Bibliotheken von Maria Stuart verzeichnen ca. 300 Bücher: DURKAN, Library, S. 92. Katharina von Medici verfügte über 4500 Bände und etwa 780 Manuskripte: BONNAFFE, Inventaire des meubles, S. 23. Das Inventar der Bibliothek von Rémi Belleau von 1577 nennt 105 Titel: Philippe HAMON, Jean JACQUART, Le XVI ${ }^{e}$ siècle, Paris 1997, S. 315-323. Isabella von Kastilien besaß 400 Bucher, ihre Tochter Johanna 44: Araceli GUILlAUME-ALONSO, Des bibliothèques féminines en Espagne (XVI ${ }^{e}-\mathrm{XVII}{ }^{\bullet}$ siècles), in: Dominique de COURCELLES, Carmen VAL JULIÁN $(\mathrm{Hg}$.), Des femmes et des livres. France et Espagnes, XIV ${ }^{e}-\mathrm{XVII}^{e}$ siècle, Paris 1999, S. 61-75, hier S. 65-67. Vgl. auch die in ihrem Umfang sehr unterschiedlichen Bibliotheksinventare in: Roger DOUCET, Les bibliothèques parisiennes au XVI siècle, Paris 1956.
} 
zeichneten Werke die Summe dessen dar, was Jahrzehnte über angeschafft wurde. Denn daß Annas Sammlung stetig wuchs, beweisen die diesbezüglich in ihren Rechnungsbüchern vermerkten Ausgaben ${ }^{38}$. So erlaubt die Bibliothek ein Blick zurück auf die vorangegangenen Jahrzehnte.

Die meisten der namentlich genannten Verfasser sind Italiener oder Franzosen, es finden sich aber auch antike Autoren, drei Deutsche sowie ein Byzantiner $^{39}$. Die Bücher sind zum großen Teil auf Französisch oder Italienisch, daneben las die Prinzessin lateinische Texte, und sie besaß zwei spanische Bücher nebst einem dictionarium Latino Hispanicum. Rund vierzig Werke historischer Thematik bilden die größte Abteilung der Bibliothek und spiegeln die weitgefaßte Neugier ihrer Leserin auf entfernte Zeiten und Länder wider. Die Geschichte Frankreichs ist mit den Chroniken von Jean Froissart und Enguerrand de Monstrelet, aber auch mit zeitgenössischen Schriften vertreten, wie die 1561 in Lyon erschienene Ausgabe der "Alliances généalogiques des rois de France et princes des Gaules « von Claude Paradin oder die 1579 erstmals in Paris verlegten »Grandes Annales et histoire générale de France« von François de Belleforest. Daneben finden sich die "Mémoires« von Olivier de La Marche (vielleicht die von Denis Sauvage besorgte Ausgabe von 1562) und die "Lettres historiques" von Pasquier in einer der von ihm selbst besorgten Editionen. Letztere könnten als \Zeitgeschichte bezeichnet werden, wie auch die sechs Bände traictant des guerres advenues depuis les barricades. Beachtung fand auch die Vergangenheit einiger der ursprünglich nicht zur französischen Krone gehörender Regionen: In den "Annales de Bourgogne« des Guillaume Paradin (Lyon 1566) sowie in den "Annales de Bretaigne« eines ungenannten Autors, die im Inventar als vieille impression beschrieben werden, so daß es sich um das 1514 zum ersten Mal gedruckte Werk von Alain Bouchard de Kerbouchart handeln könnte ${ }^{40}$.

Die italienische Geschichtsschreibung ist vertreten mit Guicciardinis «Storia d'Italia« und Machiavellis »Istorie fiorentine« sowie zwei Werken von Paolo Giovio, die "Vite brevemente scritte d'huomini illustri di guerra«, in italienischer Übersetzung 1554 in Florenz erschienen, und ein mit »Historia« bezeichneter Band, bei dem es sich entweder um das lateinische Original oder die Übersetzung seines großen Geschichtswerkes handeln muß. Des weiteren finden sich zwei Schriften zur Geschichte von Ferrara: Die 1570 ebendort erschienene »Historia de' principi d'Este« des herzoglichen Sekretärs Giovan

\footnotetext{
${ }^{38}$ Rechnungsbücher 1591 (Mai), 1593-I (Apr.), 1599-1606 (1. H. 1601).

${ }^{39}$ Bei den deutschen Autoren handelt es sich um Sebastian Münster, Laurentius Surius und Trithemius (Johannes Tritheim), bei dem byzantinischen Buch um die Kirchengeschichte des Calliste Xanthopoulos Nikephoros.

${ }^{40}$ Des weiteren finden sich zur französischen Geschichte die "Histoire générale des rois de France « von Bernard de Girard du Haillan, Paris 1576 oder 1584, und die »Annales et croniques de France« von Nicole Gilles, Paris 1553 (?).
} 
Battista Pigna und ein mit Historia di Alphonso di Ferara en italien bezeichnetes Buch, vielleicht Giovios "Vita di Alfonso da Este« (Florenz 1553) ${ }^{41}$. Neben weiteren Abhandlungen zur Geschichte von Spanien, Portugal, Polen und Zypern sind vor allem die Werke antiker Autoren aufgefuhrt, die meisten davon in französischer Übersetzung: geschichtliche Bücher von Herodot und Diodor von Sizilien, Caesars "Commentarii«, die 1565 bis 1572 bei Vascosan in Paris erschienene Doppelausgabe der »Moralia» und »Parallelbiographien« von Plutarch sowie die »Jüdische Archäologie« des spätantiken Autors Flavius Josephus, außerdem Vitruvs "De architectura» und Machiavellis "Discorsi sopra la prima deca di Tito Livio«. Weitere Bucher veranschaulichen daruber hinaus Annas Interesse an Weltkunde und Naturgeschichte im weitesten Sinne, wie etwa Sebastian Münsters "Cosmographia» oder ung livre des cartes et figures, bei dem es sich eventuell um die 1564 bei d'Orgerolles in Lyon verlegten »Plans, Pourtraitz et Descriptions de plusieurs villes et forteresses tant d'Europe, Asie, Afrique que des Indes et terres neuves« von Antoine du Pinet handeln könnte, außerdem die »Arte de navigar« des Pedro de Medina in der Übersetzung von Nicolas Nicolai und sogar eine Histoire des poissons en françois.

Bücher religiöser Thematik nehmen in Annas Bibliothek den zweiten Posten nach den historischen Werken ein: Bibeln, Psalter, Katechismen und Stundenbücher, letztere vor allem mit Gebetstexten zur Heiligen Jungfrau Maria, aber auch Breviere und ein Meßbuch in allen möglichen Sprachen, Formen und Größen finden sich ebenso wie Texte zur Kirchengeschichte und der »Gottesstaat" von Augustinus, der sich bereits im 15. Jahrhundert in den Bibliotheken adliger Damen wie Marie de Berry und Charlotte von Savoyen findet, dann in großen Auflagen gedruckt wurde und in den Sammlungen der Zeit sehr verbreitet war ${ }^{42}$. Die cens pseaulmes de David en françois besaß die Prinzessin gleich in doppelter Ausfuhrung. Hiermit dürfte das von Jean Poitevin besorgte /Gesangbuch ohne Noten gemeint sein, dessen erste Ausgabe, 1550 bei Nicolas Peletier in Lyon erschienen und dem Kardinal Jean de Lorraine gewidmet, in der Folge mehrere Neuauflagen erlebte, die dann wiederum dessen Neffen

\footnotetext{
${ }^{41}$ Weitere italienische Geschichtsbücher: Eine "Historia del mondo« von Giovan Tarcagnota und eine »Historia di casa Orsina« von Francesco Sansovino, bei der es sich wohl um einen Teil der »Origini e fatti delle famiglie illustri d'Italia« handelt.

${ }^{42}$ Zu Marie de Berry: Susan Groag BeLL, Medieval Women Book Owners: Arbiters of Lay Piety and Ambassadors of Culture, in: Signs 7 (1982) S. 742-768, hier S. 748. Zu Charlotte von Savoyen: Alexandre TUETEY, Inventaire des biens de Charlotte de Savoie, in: Bibliothèque de l'École des Chartes 26 (1865) S. 338-366, 423-442, hier S. 357. Zu den Manuskripten des "Gottesstaat" in franzősischen Sammlungen: A. PAULIN PARIS, Les manuscrits françois de la bibliothèque du roi, leur histoire et celle des textes allemands, anglois, hollandois, italiens, espagnols de la même collection, Bd. 1, Paris 1836, S. 19-29. Zu den gedruckten Ausgaben: Short-title Catalogue of Books printed in France and of French Books printed in other countries from 1470 to 1600 in the British Museum, London 1924, ND 1966, S. 34 u. Supplement, 1986, S. 6.
} 
Charles gewidmet waren ${ }^{43}$. Bei einigen der religiösen Gebrauchstexte in Annas Bibliothek handelte es sich um illuminierte Handschriften auf Pergament. Insgesamt finden sich fast vierzig Manuskripte in ihrer Sammlung, die, wie auch die gedruckten Werke, in verschiedenfarbige, teils vergoldete Kalbsleder oder andere feine Ledersorten, wie Velin und Maroquin, sowie Pergament und Samt gebunden waren.

Weniger gut ausgestattet war Annas Bibliothek mit literarischen Werken, nur etwa zehn Titel werden namentlich aufgefuhrt, einige davon doppelt. So ist Torquato Tassos "Gerusalemme liberata« sowohl auf Italienisch als auch in französischer Übersetzung vorhanden, und auch den »Orlando furioso« des Ludovico Ariosto gibt es in zweifacher Ausfuhrung. Des weiteren finden sich Matteo Bandellos »Novellen« sowie eine nicht weiter $\mathrm{zu}$ identifizierende spanische Übersetzung des »Libro del cortegiano« von Baldesar Castiglione. Einige zentrale Titel fehlen jedoch, und es ist schwierig festzustellen, ob sie sich in dem großen Haufen anonym zusammengebundener Drucke verstecken, ob sie verschenkt oder verliehen worden sind oder ob die Herzogin sie nie besessen hat. Das Fehlen eines bestimmten Titels in Annas Pariser Bibliothek muß natürlich nicht bedeuten, daß sie das Buch nicht kannte beziehungsweise nicht gelesen hat. Inventare stellen eine schwierige Quelle dar, da sie nur die Bücher verzeichnen, die zu einem bestimmten Zeitpunkt auf Tischen, in den Regalen oder Truhen lagen und vom Notar verzeichnet wurden. Es ist nicht unwahrscheinlich, daß gleich nach dem Tod der Herzogin einige der kostbareren Bände aus ihrer Sammlung entfernt wurden, denn wie das Beispiel der Katharina von Medici zeigt, gelangte die Hinterlassenschaft einer Fürstin nicht immer unversehrt vor die Augen der Notare ${ }^{44}$. Auch sagen Inventare nichts darüber aus, wie ein bestimmtes Werk in den Besitz seiner Leser gelangt ist, ob es überhaupt gelesen wurde und wenn $\mathrm{ja}$, wie oft, wann und $\mathrm{zu}$ welchen Gelegenheiten. Die Lesegewohnheiten einer Fürstin konnten sich im Laufe des Lebens ändern, wie das Beispiel der Mahaut d'Artois zeigt. Nach dem Tod ihres einzigen Sohnes kaufte sie nur noch zur Kontemplation geeignete Schriften, so daß ein Inventar ihrer Bücher vor allem religiöse Werke verzeichnen würde, obwohl die Gräfin zu manchen Zeiten anderer Literatur den Vorzug

${ }^{43} \mathrm{Zu}$ den »Cent Psalmes de David qui restoient à traduire en rithme françoise, traduictz par maistre Jan Poictevin, chantre de Sainte Radegonde de Poictiers«: Jeanice BROOKS, Les Guises et l'air de cour: images musicales du prince-guerrier, in: Yvonne BELLENGER (Hg.), Le mécénat et l'influence des Guise. Actes du Colloque de Joinville 1994, Paris 1997, S. 187-210, hier S. 202; Franco GLACONE, Les Lorraine et le Psautier de David, in: BELLENGER (Hg.), Mécénat et influence, S. 345-363, hier S. 350f.

${ }^{44}$ MICHAHELLES, Inventory, S. 4. Zu den Unzulänglichkeiten von Bibliotheksinventaren als historische Quelle: Roger CHARTIER, Les pratiques de l'écrit, in: Philippe ARIËs, Georges DuBY (Hg.), Histoire de la vie privée, Bd. 3: De la Renaissance aux Lumières, Paris 1986, S. 112-161, hier S. 129. 
gegeben haben mochte ${ }^{45}$. Hinzu kommt, daß sich gerade Frauen ihre Lektulre auf unterschiedliche Weise verschafften, sie erbten und borgten sich Bücher von Freundinnen, Bekannten und Verwandten oder ließen sie über ihre Agenten besorgen. Daneben wird sich die ferraresische Prinzessin auch in den gut ausgestatteten Bibliotheken ihrer Ehemänner bedient haben, schließlich hatte ein Besucher des Hôtel de Guise die librairie des Herzogs gelobt ${ }^{46}$.

Zwei Einträge in einem Inventar des Schlosses von Annecy, angefertigt zweieinhalb Jahre nach ihrem letzten Besuch in Savoyen, weisen darüber hinaus auf Schriften hin, welche die Prinzessin sicherlich besaß, wenn auch nicht unbedingt die hier erwähnten Exemplare. Beschrieben werden die Bände als ung grand livre couvert de parchemin blanc [nicht gebunden] intitulé Le premier volume, Les plus excellentz bastimentz de France und ung aultre grand livre de portraict de medallies et aultres ${ }^{47}$. Bei letzterem handelt es sich wahrscheinlich um den von Guglielmo Rovillio besorgten "Prontuario delle Medaglie«, bei dem anderen Buch um den 1576 erschienenen ersten Teil der Beschreibung französischer Schlösser von Jacques Androuet du Cerceau. Obwohl sie im Pariser Inventar nicht auftauchen, waren beide Werke wohl auch in Annas Bibliothek im Hôtel de Nemours vorhanden, schließlich kannte die Prinzessin du Cerceau, der viele Jahre für Renée de France in Montargis tätig war, persönlich. Und in dem 1581 in Lyon erschienenen zweiten Teil des Nachschlagewerks zu den Medaillen ist das Konterfei der Herzogin zu sehen, während sie selbst für ihre singolari virtù gelobt wird, le quali la fanno come un chiarissimo lume nel numero di Prencipesse di questo tempo ${ }^{48}$.

Vergleicht man die Bibliothek der Herzogin von Nemours mit denen anderer Fürstinnen und Fürsten, so fallen zunächst die offensichtlichen Lücken im Bereich der schönen Literatur auf. Zwar verzeichnet das Inventar von Annas Sammlung Texte von Tasso, Ariost und Bandello, doch vermißt man Dante und Petrarca, vor allem aber Boccaccios »Decamerone« oder doch zumindest eines der in seiner Folge in Frankreich entstandenen Werke. Schließlich han-

45 Jules-Marie RICHARD, Les livres de Mahaut comtesse d'Artois et de Bourgogne, 13021329, in: Revue des questions historiques 40 (1886) S. 235-241, hier S. 238. Ein Inventar ist nicht überliefert, dafür Rechnungen über Bücherkäufe.

${ }^{46}$ "Arches« an François de Lorraine, 2. Juli 1556, Paris, in: SAMARAN, Le Primatice, S. 188. Bei dem Besucher handelt es sich um den Kardinal Carlo Carafa. Zu den unterschiedlichen Methoden, wie Frauen sich Literatur beschafften: BroOMHALL, Book Trade, S. 26-31; BELL, Medieval Women, S. 748-750. Vgl. auch den Hinweis auf den Psalter, den Charlotte von Savoyen geborgt hatte, in: TUETEY, Inventaire des biens, S. 357.

${ }^{47}$ Inventayre des meubles et armes consistantz au chasteau de la presente ville d'Annessy pour rendre à Messieurs de la chambre des comptes de Genevois, 18. Aug. 1588, in: ADA, SA 96, 18, Nr. 6, fol. 22r.

${ }^{48}$ (Guglielmo RoviLLIO), Seconda parte del Prontuario delle Medaglie, la quale comincia da la natività del nostro Salvatore Giesu Christo, e continua infino al Christianissimo Rè di Francia e di Pologna, Henrico III. di nome, il quale al presente regna felicemente, Lyon 1581, T. 2, S. 283, 285. Eine Abb. in: PaTTANARO, Girolamo da Carpi, S. 92. 
delt es sich bei Boccaccio um den in Pariser Bibliotheksinventaren des 16. Jahrhunderts am häufigsten verzeichneten Autor, und Petrarcas Schriften standen in den Regalen sowohl adliger als auch nichtadliger Leser. Isabella von Kastilien etwa besaß mehrere Bücher von Boccaccio in verschiedenen Sprachen, außerdem Petrarcas »Triomphi«, einige Bände franzősischer Poesie und kastilischer Literatur sowie drei Ritterromane. ${ }^{49}$. In Annas Sammlung ist dagegen die zeitgenössische französische Literatur so gut wie uberhaupt nicht vertreten. Während die Bibliothek der Maria Stuart neben Werken von Rabelais, du Bellay und Ronsard auch solche von heute unbekannteren Autoren wie Olivier de Magny und Marc Claude de Buttet enthielt, fehlen derartige Titel im Inventar des Hôtel de Nemours gänzlich. Dabei gehörte Ronsard zu den populärsten Lyrikern seiner Zeit, und allein im 16. Jahrhundert erschienen über zwanzig verschiedene Editionen seiner Werke, von denen in den meisten Bibliotheksinventaren mindestens ein Exemplar verzeichnet ist ${ }^{50}$. Es ist möglich, daß Anna diese Bücher besaß, sie aber nie hat binden lassen. Die Schriften würden demnach zu dem großen Haufen der im Inventar zwar erwăhnten, namentlich aber nicht erfaßten Werke zählen, doch würde auch dies die eher geringe Wertschätzung ihrer Besitzerin fur diese Art von Literatur verdeutlichen.

Merkwürdig ist überdies das Fehlen eines der beliebten Ritterromane, vor allem aber des »Amadís de Gaula« des Spaniers Garci Ordoñez de Montalvo. Die Übersetzung der ersten acht Bücher dieses Werkes durch Nicolas Herberay des Essarts erlebte in Frankreich allein Anfang der 1540er Jahre kurz hintereinander zwölf Auflagen, ein Erfolg, der sich mit den folgenden Büchern wiederholte, so daß der "Amadís « bis zum Ende des 16. Jahrhunderts zu den meistgelesenen Erzählungen der Zeit gehörte und in den Bibliotheken des europäischen Adels fast immer vorhanden war. Sum bukes of Amadis in Spa-

${ }^{49} \mathrm{Zu}$ Boccaccio: Alexander H. SCHUTZ, Vernacular Books in Parisian Private Libraries of the Sixteenth Century According to the Notarial Inventories, Chapel Hill 1955, S. 26, 37f. Zu Petrarca in den Bibliotheken der Condé und des Claude Bellièvre: Émile CHATELAIN, Recherche sur les plus vieux livres des Condé, in: Mélanges offerts à M. Émile Picot, membre de l'Institut, par ses amis et ses élèves, Bd. 2, Paris 1913, S. 487-504, hier S. 501, 503; Lucien AUVRAY, La bibliothèque de Claude Bellièvre (1530), in: Mélanges Picot, S. 333363, hier S. 350, 357. Zu Isabella von Kastilien: GunlaUME-ALONSO, Bibliothèques féminines, S. 68f.

${ }^{50} \mathrm{Zu}$ Maria Stuart: Inventar der Bibliothek des Schlosses von Edinburgh, 1578, in: SHARMAN, Library, S. 59, 67, 86, 98, 101, 103, 106, 111f. Zur Bedeutung von Ronsard: SCHUTZ, Vernacular Books, S. 21f., 67. Ronsards Werke standen in den Bibliotheken der Condé und der Montmorency sowie in der des Claude Chappuis: Chatelain, Livres des Condé, S. 493; MIROT, Montmorency, Bd. 79, S. 382, Bd. 80, S. 153; Henri OMONT, Inventaire de la bibliothèque de Claude Chappuis, libraire du roy (1575), in: Bulletin de la Société de l'histoire de Paris et de l'Ile-de-France 15 (1888) S. 22-26, hier S. 24. Vgl. Pollie BROMILOW, Les femmes lectrices de la fiction au seizième siècle. Enjeux idéologiques d'un lieu commun, in: Isabelle BROUARD-ARENDS (Hg.), Lectrices d'Ancien Régime, Rennes 2003, S. 261-268. 
nish standen in den Regalen der schottischen Königin, während in den Sammlungen der franzősischen Hauptstadt zahlreiche Ritterepen um Artus und Merlin, Parzival und Tristan zu finden waren ${ }^{51}$. Bedenkt man schließlich die Erziehung, welche Anna in Ferrara durch ihre Mutter erhalten hatte, so vermißt man noch einen weiteren Posten unter den Büchern ihrer Bibliothek: die Literatur von Frauen, speziell für Frauen oder aber über Frauen. So war etwa in der Bibliothek der Charlotte von Savoyen, Gemahlin Ludwigs XI., das "Livre des Trois Vertus « der Christine de Pizan zu finden, während der Konnetabel Charles de Bourbon gleich drei Exemplare der »Cité des dames« besaß, die auch in den Sammlungen nichtadliger Bücherbesitzer vorhanden war ${ }^{52}$. Isabella von Kastilien besaß sechs Bände, die der Querelle des femmes zugeordnet werden können, in der Bibliothek der schottischen Königin in Edinburgh befand sich eine franzősische Übersetzung der "Institutio feminae christianae» des Juan Luis Vives, und das Inventar der Sammlung der Luise von Lothringen im Schloß von Chenonceaux verzeichnet ein mit »les Femmes Illustres" betiteltes Buch, bei dem es sich um Boccaccios "De claris mulieribus« handeln könnte ${ }^{53}$.

Nicht minder erstaunlich ist auf der anderen Seite die Häufigkeit historischer Bücher, die Anna in noch größerer Zahl besessen hat als solche religiöser Thematik. Verglichen mit den Sammlungen anderer Fürstinnen der Zeit ist dies eine echte Seltenheit. So ähnelt beispielsweise die Bibliothek der Diane de Poitiers in ihren thematischen Schwerpunkten der des Hôtel de Nemours, auch in ihr finden sich vor allem Bücher zur Geschichte, ferner zu Naturgeschichte, Geographie und Literatur, doch nehmen religiöse Werke den ersten Platz vor allen anderen ein, und auch unter den Manuskripten der Katharina von Medici stellt die "Theologica graeca» den umfangreichsten Posten dar ${ }^{34}$.

${ }^{51}$ Zur großen Beliebtheit des "Amadís« im Frankreich des 16. Jh. und zu den Ritterromanen: Eugène BARET, De l'Amadis de Gaule et de son influence sur les mours de la littérature au $\mathrm{XVI}^{e}$ et au XVII ${ }^{e}$ siècle, Paris ${ }^{2} 1873$, ND 1970, S. 164-175; SCHUTZ, Vernacular Books, S. 32, 54, 57, 62, 70f. Zu Maria Stuart: Inventar der Bibliothek des Schlosses von Edinburgh, 1578, in: SHARMAN, Library, S. 37, 71. Das Zitat: DURKAN, Library, S. 102.

${ }^{52}$ ZIMMERMANN, Salon, S. 78; Inventar der Bibliothek der Charlotte von Savoyen, 1484, in: TUETEY, Inventaire des biens, S. 359. Zu Charles de Bourbon: Inventar der Bibliothek des Schlosses von Moulins, 1523, in: FRANCE, Enseignements, S. 238, 252, 256. Zu den nichtadligen Sammlern: SCHUTZ, Vernacular Books, S. 41; Alexandre VIDIER, Un bibliophile du $\mathrm{XVI}^{\boldsymbol{e}}$ siècle: Nicolas Moreau, $\mathrm{S}^{\mathrm{r}}$ d'Auteuil, in: Mélanges offerts à $\mathrm{M}$. Émile Picot, membre de l'Institut, par ses amis et ses élèves, Bd. 2, Paris 1913, S. 371-375, hier S. 374.

${ }^{53}$ ZIMMERMANN, Salon, S. 105; Inventar der Bibliothek Isabellas von Kastilien, 1504, in: GUILLAUME-ALONSO, Bibliothèques féminines, S. 68f.; Inventar der Bibliothek des Schlosses von Edinburgh, 1578, in: SHARMAN, Library, S. 73; Inventar des Schlosses von Chenonceaux, 1603, in: Augustin GALITZIN, Inventaire des meubles, bijoux et livres estant à Chenonceaux le huit janvier MDCIII, précédé d'une histoire sommaire de la vie de Louise de Lorraine, reine de France, Paris 1856, S. 14.

${ }^{54}$ Katalog der Bücher, die mit einiger Sicherheit Diane de Poitiers gehört haben, in: Jean PORCHER, Les livres de Diane de Poitiers, in: Les trésors des bibliothèques de France, 
Außer dem Schwergewicht weltlicher Titel ist in Annas Sammlung die Vielfalt der in den Werken behandelten historischen Themen erstaunlich. Neben Büchern zur Geschichte Frankreichs und Italiens, die sich fast allen Epochen von der Antike bis zu den jüngsten Ereignissen widmen, finden sich Abhandlungen über viele andere Länder sowie über einzelne Regionen und Fürstenhäuser. Der Vergleich mit der Bibliothek der Königin von Schottland, die ebenfalls eine ganze Reihe von Geschichtsbüchern enthielt, die inhaltlich aber über die auch im Hôtel de Nemours vorhandenen Standardwerke von Froissart, Monstrelet und Paradin kaum hinausgehen, verdeutlicht, wie außergewöhnlich die Sammlung der Prinzessin in dieser Hinsicht war ${ }^{55}$.

Nicht nur Anzahl und Inhalt der Bücher in Annas Bibliothek, auch ihr materieller Wert ist beträchtlich, denn er umfaßt mit 280 Livres über sechs Prozent der gesamten Hinterlassenschaft, vergleichbar mit der Pariser Bibliothek von Vincenzo Ludovisi, welcher immerhin einen großen Teil seines Vermögens in den Kauf von Büchern investierte ${ }^{56}$. Die meisten der Stücke sind bei der Inventarisierung auf weniger als einen Écu geschätzt worden, gut zwei Dutzend von ihnen waren jedoch mehr wert, darunter drei der Bibeln zu je acht oder neun Livres. Den kostbarsten Band stellte ein Foliant dar, beschrieben als les grandz bréviaires du feu roy en deux temps, reliéz en maroquin noir, priséz vingt quatre livres $t$. So weisen Umfang, Wert und Inhalt der Sammlung Anna als eine Dame aus, die ihre Bildung nicht als mit dem Ende ihrer Erziehung in Ferrara abgeschlossen ansah, sondern ihr Interesse für Geschichte und Geographie, für italienische und französische Autoren das ganze Leben lang kultivierte.

Bd. 25, Paris 1942, S. 78-89, hier S. 80-86; Auswertung des Inventars des Hôtel de la Reine in Paris mit den Manuskripten der Katharina von Medici, in: Emest QUENTIN-BAUCHART, La bibliothèque de Fontainebleau, 1515-1589. Étude historique et bibliographique des collections de livres manuscrits et imprimés des derniers Valois, Paris 1891, ND 1970, S. 182-185: Unter den 776 Einträgen finden sich 203 theologische Werke und 61 historische.

${ }^{55}$ Inventare der Bibliotheken der Schlösser von Holyrood, 1569, und Edinburgh, 1578, in: SHARMAN, Library, S. 44, 81, 136, 141f. u. DURKAN, Library, S. 102.

${ }^{36}$ Vgl. DuBost, France italienne, S. 291f. Vincenzo Ludovisi war Sekretär von Concino Concini. Für seine Bibliothek (1629) war ein Wert von 146 l.t. berechnet worden, was $9 \%$ des gesamten Vermögens entspricht. 